\title{
Valvoplastia Aórtica Percutânea em Adolescente Gestante
}

\author{
Rogério T. Tumelero, Norberto T. Duda, Alexandre P. Tognon, Iselso Sartori, Silvane Giongo \\ Passo Fundo, RS
}

Relatamos um caso em que a valvoplastia aórtica percutânea foi utilizada como primeira escolha, em procedimento de urgência, para o tratamento de estenose aórtica grave em paciente gestante de 16 anos, com congestão pulmonar sem controle clínico. Descrevem-se o quadro clínico, a fisiopatologia, os aspectos diagnósticos e indicações do tratamento percutâneo.

A estenose valvar aórtica congênita, quando grave, é rara em crianças e jovens. A valva aórtica bicúspide ocorre em $3 \%$ a $6 \%$ com doença cardíaca congênita e, quando relacionada com fusão comissural, pode haver estenose importante já na infância. A associação de estenose aórtica congênita grave com gestação é de difícil controle clínico e alto risco de mortalidade materna e fetal, principalmente quando se manifesta com sintomas de congestão pulmonar ${ }^{1,2}$.

\section{Relato de Caso}

Paciente gestante, 16 anos, branca, 27 semanas de gestação, interna com quadro de dispnéia intensa e dor precordial. Como antecedentes pessoais, sopro cardíaco sem diagnóstico ou acompanhamento. Ao iniciar o pré-natal, há duas semanas da internação, foi encaminhada para avaliação cardiológica, com queixas de cansaço aos esforços. Evoluiu com sintomas ao repouso, sendo internada. Referia tabagismo, sem história familiar de cardiopatia, diabetes melittus, hipertensão ou dislipidemia.

Ao exame físico, regular estado geral, taquipnéica, acianótica, fácies incaracterística, peso de $40 \mathrm{Kg}$ e estatura de $1,50 \mathrm{~m}$. Ausculta cardíaca com ritmo regular, taquicardia (freqüência cardíaca $=120 \mathrm{bpm}$ ) e pressão arterial $=90 \times 60$ mmHg, sopro sistólico de ejeção 5+/6+ no foco aórtico com irradiação para artérias carótidas. Ausculta pulmonar com estertores crepitantes bibasais.

Faculdade de Medicina da Universidade de Passo Fundo e Hospital São Vicente de Paulo Correspondência: Rogério Tadeu Tumelero - Rua Teixeira Soares, 777/sala 705 Cep 99010-080 - Passo Fundo, RS - e-mail: rttumelero@terra.com.br

Recebido para publicação em 4/10/02

Aceito em 14/1/03
Ao eletrocardiograma, ritmo sinusal, sobrecarga das câmaras esquerdas e alterações secundárias à sobrecarga na repolarização ventricular(fig. 1).

Radiografia de tórax, área cardíaca normal, diversão cranial da circulação pulmonar, linhas septolinfáticas B de Kerley, compatíveis com congestão pulmonar.

Ao ecocardiograma na admissão, valva aórtica bicúspide com fusão comissural da cúspide coronariana direita e esquerda, mobilidade sistólica moderadamente reduzida, dinâmica sistólica em cúpula e ausência de calcificação. Hipertrofia concêntrica do ventrículo esquerdo (VE), gradiente ventrículo esquerdo/aorta $(\mathrm{VE} / \mathrm{AO})=100 \mathrm{mmHg}$, anel aórtico medindo $1,48 \mathrm{~cm}^{2}$.

Mantida em tratamento clínico na unidade coronariana, em uso de digitálico, diurético, nitroglicerina e oxigenoterapia por máscara.

Oultra-som obstétrico, na admissão, demonstrou batimentos cardíacos e movimentos fetais presentes, placenta grau 0 de Grannun, com espessura normal. Líquido amniótico com volume normal. Peso aproximado $920 \mathrm{~g}$, sem particularidades da morfologia fetal. Biometria fetal média para a idade gestacional ecográfica de 27 semanas. Realizado novo ultra-som a cada $48 \mathrm{~h}$ para acompanhamento do feto.

$\mathrm{Na} 30^{\circ}$ semana de gestação a gestante evoluiu com

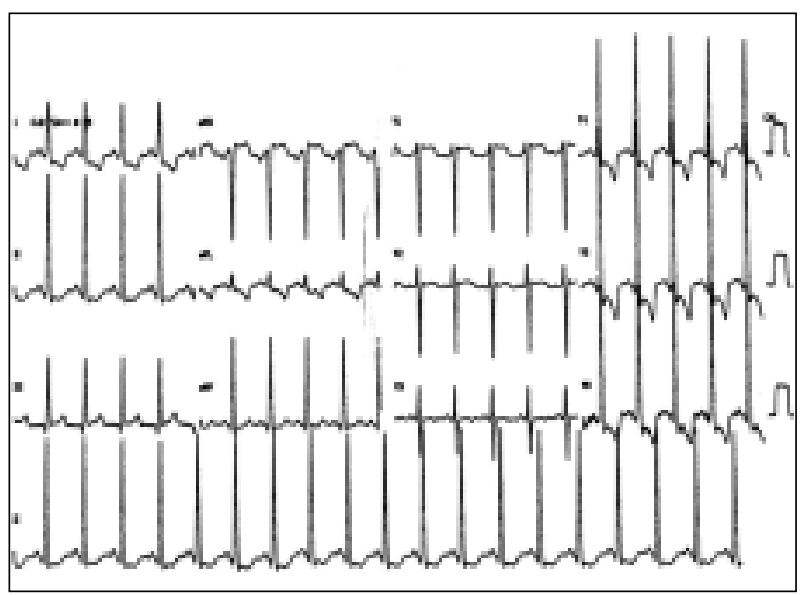

Fig. 1 - ECG de repouso na admissão hospitalar demonstrando sobrecarga de câmaras esquerdas. 
congestão pulmonar progressiva, com insuficiente resposta ao tratamento clínico, sendo indicado valvoplastia aórtica percutânea.

Ao cateterismo cardíaco, valva aórtica bicúspide com gradiente $\mathrm{VE} / \mathrm{AO}=105 \mathrm{mmHg}$ (fig. 2), aorta ascendente com aspecto angiográfico normal, circulação coronariana com origem e trajeto normais, ventrículo esquerdo hipertrófico.

Procedimento - paciente sedada, com monitorização dos batimentos cardiofetais. Cateterização do ventrículo esquerdo com cateter Sones 8F, através de punção da artéria femoral direita. Posicionado guia 0.35 (Amplatz "extra-stiff" $260 \mathrm{~cm}$ - Boston Scientific) no ventrículo esquerdo. Aortografia em projeção oblíqua anterior esquerda e ventriculografia esquerda em projeção oblíqua anterior direita (cateter angiográfico 5F Multi-Track-NuMed Inc.) com contraste radiográfico não iônico de baixa osmolaridade. Utilizado balão 18x4 cm (Z-MED - NuMed) (fig. 3). Ao término do procedimento, gradiente de ejeção pico-a-pico VE/AO de $20 \mathrm{mmHg}$ e insuficiência valvar aórtica de grau discreto a moderado (fig. 4).

No eletrocardiograma pós-procedimento houve redução da frequiência ventricular e manutenção dos sinais de sobrecarga das câmaras esquerdas.

A radiografia de tórax demonstrou redução dos sinais de congestão pulmonar, e o ecocardiograma estenose aórtica residual de grau discreto (gradiente $\mathrm{VE} / \mathrm{AO}=29 \mathrm{mmHg}$ ), insuficiência valvar aórtica de grau moderado, demais aspectos inalterados.

Após o procedimento foi realizada monitorização fetal através da cardiotocografia, que apresentou traçado reativo. A ecografia e a dopplervelocimetria colorida obstétricas demonstraram boa vitalidade fetal. No $4^{\circ}$ dia pós valvoplastia aórtica percutânea realizou-se nova ecografia obstétrica, observando-se oligodramnia grave sendo ILA (índice de lí-

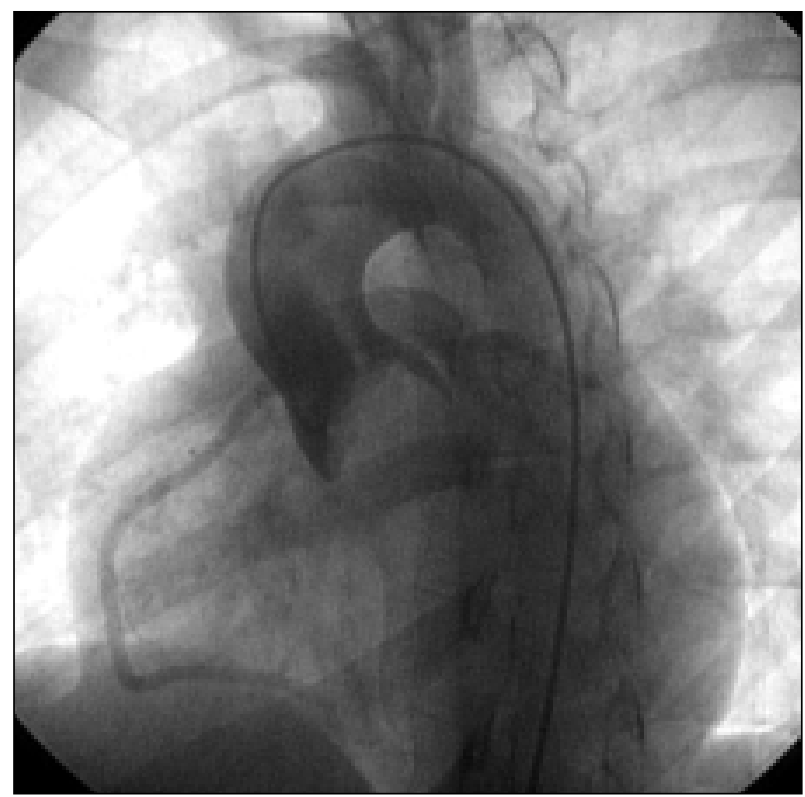

Fig. 2 - Aortograma na incidência oblíqua anterior esquerda. Observa-se valva aórtica bicúspide com estenose grave (diminuição da opacidade indicando fluxo pela valva estenosada).

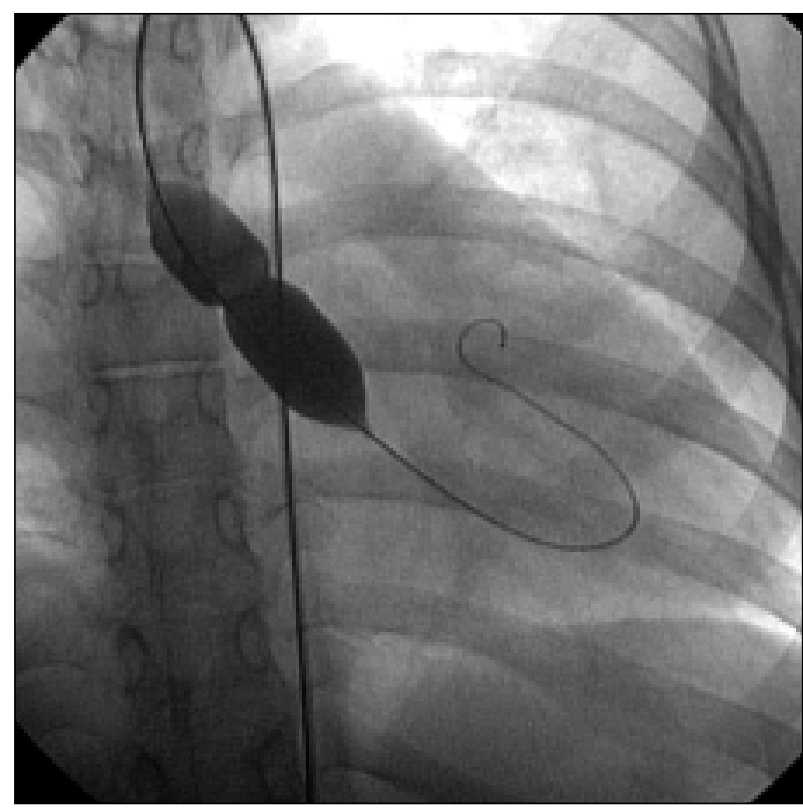

Fig. 3 - Aortograma na incidência oblíqua anterior direita. Dilatação da valva aórtica com balão. Observa-se constrição correspondente à estenose valvar.

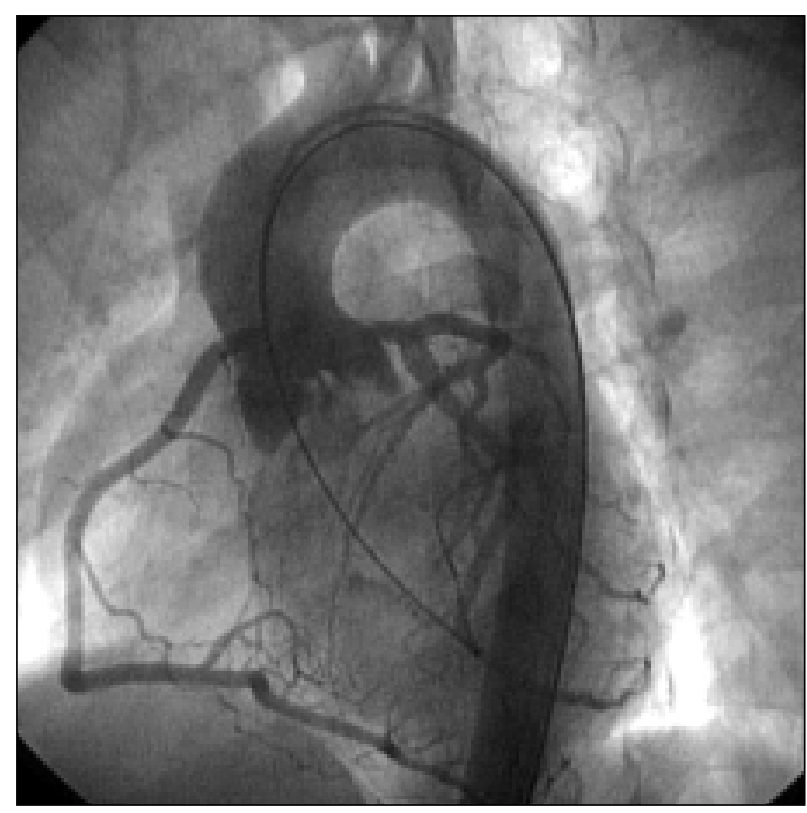

Fig. 4 - Aortograma na incidência oblíqua anterior esquerda. Resultado final demonstrando discreta regurgitação aórtica e desaparecimento do jato central.

quido amniótico $)=2,5 \mathrm{~cm}$ (normal $8-18 \mathrm{~cm})$. Devido à associação de oligodramnia com insuficiência placentária foi indicada interrupção da gestação com parto cesariano.

Orecém nascido era do sexo masculino, pesando $1.425 \mathrm{~g}$, escala de Apgar 8e 9 eíndice de Capurro de 32 semanas.

Alta hospitalar materna no $4^{\circ}$ dia após a cesariana, em bom estado geral, sem dispnéia. O diurético e o inibidor da enzima de conversão da angiotensina foram mantidos para controle clínico da insuficiência cardíaca.

$O$ recém nascido, prematuro, apresentou quadro de membrana hialina, broncopneumonia extensa, septicemia, 
coagulação intravascular disseminada e anemia. Além disso, mantinha a presença do canal arterial patente, sendo utilizada indometacina até seu fechamento, com 10 dias de vida. Recebeu alta hospitalar com 46 dias de vida, em bom estado geral, estável e com peso de $2.125 \mathrm{~g}$.

No seguimento clínico de 9 meses a paciente permaneceu assintomática, em uso de inibidor da enzima de conversão da angiotensina e diurético. Devido à boa evolução clínica, foi postergada a correção cirúrgica valvar.

\section{Discussão}

A estenose valvar aórtica congênita, quando grave, é rara em crianças e jovens ${ }^{1}$. A valva aórtica bicúspide corresponde de $3 \%$ a $6 \%$ das doenças cardíacas congênitas ${ }^{1,2}$. Ocorre mais freqüentemente em homens, na razão de 4:1, e associação de anomalias cardiovasculares pode atingir $20 \%{ }^{2}$. O trauma produzido pela turbulência do fluxo sangüíneo leva ao espessamento, fibrose, calcificação e rigidez, com as manifestações clínicas ocorrendo, então, a partir da $3^{\circ}$ década de vida, predominantemente no sexo masculi$\mathrm{no}^{2,3}$. Em alguns casos, quando a estenose valvar bicúspide relaciona-se à fusão comissural, pode se apresentar grave já na infância ${ }^{3}$. Alguns autores a consideram a mais freqüente das cardiopatias congênitas, uma vez que não costuma ser detectada nos primeiros anos de vida. Com o advento da utilização rotineira de técnicas ecocardiográficas houve facilitação do seu reconhecimento ${ }^{2}$.

As alterações fisiológicas induzidas pela gestação impõem três alterações hemodinâmicas maiores ao coração: aumento do débito cardíaco (30-40\%); aumento da freqüência cardíaca (10-20bpm)e a expansão do volume sangüíneo (20-100\%) $)^{4,5}$. A associação desses fatores com a obstrução da via de saída do ventrículo esquerdo que, por sua vez, limita as variações do débito cardíaco, podem levarà descompensação hemodinâmica, freqüentemente traduzida por sintomas e sinais de congestão pulmonar, síncope e morte súbita.

Segundo Arias e Pineda ${ }^{6}$, em uma série de 38 gestações com 23 estenoses aórticas graves, a história natural dessa doença determina mortalidade materna de $17,4 \%$ no período de gestação das pacientes não tratadas e mortalidade fetal de $34 \%$. Uma intervenção cardíaca invasiva pode ser necessária em pacientes com deterioração do seu estado clínico durante a gravidez, com o objetivo de reduzir o gradiente de ejeção pico-a-pico em $60 \%$ a $70 \%{ }^{7,8}$. O tratamento cirúrgico da estenose aórtica bicúspide grave é recomendado pelo Consenso Brasileiro Sobre Cardiopatia e Gravidez em qualquer época da gestação quando o gradiente VE/AO for maior que $70 \mathrm{mmHg}^{9}$. Cheitlin e cols ${ }^{10}$ recomendam valvoplastia cirúrgica com balão ou ainda a substituição cirúr- gica valvar quando tiver sintomas, prontamente se houver evidências de congestão pulmonar, e que tenha área valvar de $0,7 \mathrm{~cm}^{2}$ ou menor, medidos no ecocardiograma ou no cateterismo cardíaco. A valvoplastia por balão, utilizada com sucesso em estenose mitral grave na gravidez e em estenoses aórticas em pacientes não grávidas, mas de alto risco para substituição da valva aórtica ${ }^{11,12}$, foi aplicada em duas pacientes grávidas com estenose aórtica ${ }^{13,14}$. Ambos os casos tiveram desfechos maternos e fetais favoráveis. Uma série de valvoplastias realizadas em gestações na $30^{\mathrm{a}}$ sema$\mathrm{na}^{15}$ foi associada com parto pretermo duas semanas mais tarde de bebes saudáveis. Em dois estudos ${ }^{16,17}$ não houve óbitos maternos em 11 pacientes com substituição valvar aórtica apesar da mortalidade cirúrgica global materna com derivação cardiopulmonar ter sido de 1,5. Em contraste, a mortalidade cirúrgica fetal global variou entre $16 \%{ }^{17} \mathrm{e}$ $20 \%{ }^{18}$. A substituição valvar aórtica, em particular, parece estar associada à mortalidade fetal excepcionalmente alta, $40 \%$ em todo o grupo e $57 \%$ em pacientes com estenose aórtica ${ }^{17}$. Dessa forma, a cirurgia aberta para estenose valvar aórtica durante a gravidez deva ser considerada, provavelmente, como o último recurso.

A valvoplastia aórtica percutânea por balão teve seu início em meados dos anos 80. A partir da experiência inicial vivida na valvoplastia pulmonar, Lababidi ${ }^{19}$ e Lababidi e $\mathrm{Wu}^{20}$, em 1984, estenderam o método para o tratamento dessa anomalia. Atualmente, o aperfeiçoamento dos materiais propiciou a utilização desse método, menos invasivo, nas situações em que o risco cirúrgico é inaceitável.

A valvoplastia com balão, bem como a valvoplastia cirúrgica, são limitadas pelos seus resultados tardios, conferindo a esses procedimentos o status de tratamento paliati$\mathrm{vo}^{21}$. O surgimento imediato de regurgitação aórtica ou sua progressão e, tardiamente, a reestenose, são as maiores complicações da valvoplastia. Outras complicações, durante o procedimento incluem sangramentos, arritmias, acidente vascular cerebral, complicações arteriais ilíaco-femoral, dano à valva mitral e a morte ${ }^{7}$.

Outro cuidado é a exposição aos raios $\mathrm{X}$ durante o procedimento, que por um período curto de tempo, pode ser minimizada utilizando-se proteção radiológica para o abdome e pelve da gestante, reduzindo ao máximo os risco de malformações congênitas ${ }^{22}$.

A experiência com valvoplastia aórtica percutânea em gestantes limita-se a relatos de caso, já que é realizada nas pacientes instáveis clinicamente necessitando de intervenção e com risco cirúrgico materno-fetal elevado. A valvoplastia aórtica percutânea durante a gestação é um procedimento seguro e efetivo, reduzindo a morbimortalidade e dando oportunidade a uma correção valvar cirúrgica eletiva. 


\section{Referências}

1. Roberts, WC. The structure of the aortic valve in clinically isolated aortic stenosis an autopsy study of 162 patients over 15 years of age. Circulation 1970; 41(C):91

2. Friedman WF. Congenital Heart Disease in Infancy and Childhood. In Braunwald E (ed) Heart Disease: A Textbook of Cardiovascular Medicine. $5^{\text {th }}$ ed. Philadelphia: WB Saunders Co, 1997; 29:877-962.

3. MartinezEE, Portugal OP. Valvopatia Aórtica. EmBarreto ACP, Souza AGMR(eds). Cardiologia Atualização e Reciclagem. Rio de Janeiro, Ateneu, 1994;44:447-54.

4. Elkayam U, GleicherN. Hemodynamics and cardiac function during normal pregnancy and the puerperium. In Elkayam U, Gleicher N (eds): Cardiac problems in pregnancy: diagnosis and management of maternal and fetal disease. $2^{\text {nd }}$ ed. New York: Alan R. Liss, Inc, 1990, p5.

5. Machini IS, Albazzaz SJ, Fadel HE, et al. Serial noninvasive evaluation of cardiovascular hemodynamics during pregnancy. Am J Obstet Gynecol 1987;156: 1808-12.

6. Arias F, Pineda J. Aortic stenosis and pregnancy. J Reprod Med 1978;20:229-32

7. McCrindle BW for the Valvuloplasty and Angioplasty of Congenital Anomalies (VACA) Registry Investigators. Independent predictors of immediate results of percutaneous balloon aortic valvotomy in childhood. Am J Cardiol 1996;772:286

8. Sandhu SK, Silka MJ, Reller MD. Balloon aortic vasvuloplasty for aortic stenosis in neonatos, children and young adults. I Interven Cardiol 1995;8:477.

9. Consenso Brasileiro Sobre Cardiopatia e Gravidez. Arq Bras Cardiol 1999;72(supIII):5-26

10. Cheitlin MD. The Timing of surgery in mitral and aortic valve disease. Curr Probl Cardiol 1987;12:112-23.
11. Letac B, Cribier A, Konin R, et al. Results of percutaneous transluminal valvoplasty in 218 adults with valvular aortic stenosis. Am J Cardiol 1988; 62:598-605.

12. Kuntz RE, Tosteson ANA, Berman A, et al. Predictor of event-free survival after balloon aortic valvuloplasty. N Engl J Med 1991;325:17-23.

13. Angel JL, Chapman C, Knuppel RA, et al. Percutaneous balloon valvoplasty in pregnancy. Obstet Gynecol 1988:3:438-40.

14. McIvor RA. Percutaneous balloon aortic valvoplasty during pregnancy. Int J Cardiol 1991;32:1-4.

15. Colclough G. Epidural anesthesia for cesarean delivery in a parturient with aortic stenosis. Reg Anesthe 1990;15:273-4

16. Ben-Ami M, Battino S, Rosenfeld T, et al. Aortic valve replacement during preg nancy. Acta Obstet Gynecol Scand 1990;69:651-3.

17. BeckerRM. Intracardiac surgery in pregnant women. Ann Thorac Surg 1983;36:453-8

18. Bernal JM, Miralles PJ. Cardiac surgery with cardiopulmonary bypass during pregnancy. Obstet Gynecol Surg 1986;41:1-6.

19. Lababidi Z. Aortic balloon valvuloplasty. Am Heart J 1983;106:751-5.

20. Lababidi Z, Wu JR. Percutaneous balloon aortic valvuloplasty: results in 23 patients. Am J Cardiol 1984;53:194-7.

21. Fontes VF, Esteves CA, Braga SLN, et al. Cateterismo intervencionista das cardiopatias congênitas. In Barreto ACP, Souza AGMR (eds). Cardiologia Atualização e Reciclagem. Rio de Janeiro: Ateneu, 1994;57:595-619.

22. Dosimetry Working Party of the Institute of Physical Sciences in Medicine. National Protocol for patient dose measurements in diagnostic radiology. Oxford: National Radiation Protection Board, 1992:14. 